\title{
$\mathrm{C} / \mathrm{C}$ 复合材料拉伸强度与针刺成型参数相关性研究
}

\author{
郑金煌 ${ }^{1}$, 李贺军 ${ }^{1}$, 崔 红 ${ }^{1,2}$, 王 毅 ${ }^{2}$, 邓海亮 ${ }^{2}$, 殷忠义 ${ }^{2}$, \\ 姚冬梅 ${ }^{2}$, 苏 红 ${ }^{2}$
}

(1. 西北工业大学 凝固技术国家重点实验室, 西安 710072; 2. 西安航天复合材料研究所, 西安 710025)

摘 要: 采用 Design-expert 软件设计预制体不同针刺成型参数组合试验, 研究预制体针刺成型参数对针刺碳/碳(C/C) 复合材料拉伸强度的影响, 并构建了响应曲面数学模型, 实现对针刺 $\mathrm{C} / \mathrm{C}$ 复合材料拉伸强度的优化与预测, 其模型 显著性 $P=0.0206$, 各试验实测值与预测值相对误差 $\leqslant 10.82 \%$, 模型具有较高的拟合度。响应曲面回归分析表明: 针 刺深度对拉伸强度有极显著影响, 针刺密度对拉伸强度有显著影响, 在本研究的针刺成型参数取值范围内, 拉伸 强度的预测区间为 42.31 91.87 MPa。通过模型优化出的针刺成型参数组合为: 针刺密度 $11 \mathrm{pin} / \mathrm{cm}^{2}$ 、针刺深度 $11 \mathrm{~mm}$ 、网胎面密度 $50 \mathrm{~g} / \mathrm{m}^{2}$, 相应拉伸强度预测值为 $88.62 \mathrm{MPa}$, 验证值为 $90.71 \mathrm{MPa}$ ，相对误差 $2.36 \%$ 。

关 键 词: 针刺预制体; 碳/碳复合材料; 拉伸强度; 响应曲面; 模型

中图分类号: TB332 文献标识码: A

\section{Relations between Needling Processing Parameters and Tensile Strength of C/C Composites}

\author{
ZHENG Jin-Huang ${ }^{1}$, LI He-Jun ${ }^{1}$, CUI Hong ${ }^{1,2}$, WANG Yi $^{2}$, DENG Hai-Liang ${ }^{2}$, \\ YIN Zhong- $\mathrm{Yi}^{2}$, YAO Dong-Mei ${ }^{2}$, SU Hong ${ }^{2}$
}

(1. State Key Laboratory of Solidification Processing, Northwestern Polytechnical University, Xi'an 710072, China; 2. Xi'an Aerospace Composite Research Institute, Xi'an 710025, China)

\begin{abstract}
The experiments for the fabrication of needling preform were designed according to response surface methodology (RSM) by the design-expert software to study effects of three variable needling processing parameters on the tensile strength of carbon/carbon (C/C) composites. Mathematical model between needling processing parameters and tensile strength of $\mathrm{C} / \mathrm{C}$ composites was set up to optimize the needling processing parameters of preform and to predict the tensile strength of $\mathrm{C} / \mathrm{C}$ composites. The mathematical model has good fitting degree with $\mathrm{P}$-value of 0.0206 and the relative error between the predicted and the actual is no more than $10.82 \%$. Based on the analysis of response surface of tensile strength, the needling depth has very significant influence and the needling density has significant influence on the tensile strength of $\mathrm{C} / \mathrm{C}$ composites. The predicted scope of the tensile strength by the mathematical model is from 42.31 MPa to 91.87 MPa. The calculated optimal values are tensile strength $88.62 \mathrm{MPa}$, needling density $11 \mathrm{pin} / \mathrm{cm}^{2}$, needling depth $11 \mathrm{~mm}$, and density of non-woven $50 \mathrm{~g} / \mathrm{m}^{2}$, while the corresponding experimental result is $90.71 \mathrm{MPa}$ with relative error $2.36 \%$.
\end{abstract}

Key words: needling preform; carbon/carbon composites; tensile strength; response surface methodology; model

收稿日期: 2017-01-22; 收到修改稿日期: 2017-03-19

基金项目：国家 973 计划(613184); 国家自然科学基金(51202233); 装备预研基金(9140A12060514HT43190)

National Basic Research Program of China (973Program 613184); National Natural Science Foundtion of China (51202233); Equipment Research Foundation (9140A12060514HT43190)

作者简介: 郑金煌(1973-), 男, 高级工程师. E-mail: zjh114@sohu.com

通讯作者: 李贺军, 教授. E-mail: lihejun@nwpu.edu.cn; 崔 红, 研究员. E-mail: cuihong@ht43.com.cn 
碳纤维预制体作为增强体, 对碳/碳(C/C)复合材 料的性能起到决定性作用 ${ }^{[1]}$ 。针刺成型碳纤维预制体 (针刺预制体)具有孔隙分布均匀、易致密成型等优点, 并且克服了二维铺层结构层间强度低、编织结构预 制体工艺复杂及成本高等缺点 ${ }^{[2-4]}$, 成为美、法等发 达国家优先发展的一种增强预制体成型技术 ${ }^{[5-11]}$ 。

拉伸强度是针刺 $\mathrm{C} / \mathrm{C}$ 复合材料应用的一个核心 技术指标，直接反映了针刺预制体的面内性能。不 同的应用工况需要研究预制体成型工艺与 $\mathrm{C} / \mathrm{C}$ 复合 材料性能的关联性 ${ }^{[12]}$, 以便对针刺 $\mathrm{C} / \mathrm{C}$ 复合材料拉 伸性能进行可控设计和预测。然而, 由于针刺预制 体性能表征的难度较大、 $\mathrm{C} / \mathrm{C}$ 复合材料的成型工艺 波动大、性能离散不可避免, 以及多个针刺成型参 数间的交互作用复杂, 导致针刺预制体及针刺 $\mathrm{C} / \mathrm{C}$ 复合材料的性能设计与预测难度较大, 进而制约了 针刺 $\mathrm{C} / \mathrm{C}$ 复合材料的性能发挥与拓展应用。科学家 研究了针刺预制体成型参数对 $\mathrm{C} / \mathrm{C}$ 复合材料力学性 能的影响 ${ }^{[2-17]}$, 并获得了针刺密度、针刺深度、网 胎面密度等影响拉伸强度的关键针刺成型参数。但 相关研究主要集中在单个针刺成型参数对针刺预制 体或针刺 $\mathrm{C} / \mathrm{C}$ 复合材料性能的定性影响, 并未研究 针刺成型参数之间的交互作用, 特别是未构建出二 者的相关性模型, 无法对针刺 $\mathrm{C} / \mathrm{C}$ 复合材料拉伸性 能进行量化设计与预测。基于此, 本工作运用数理 统计与实验研究相结合的方法, 以针刺密度、针刺 深度、网胎面密度等针刺成型参数为关键影响因素, 针刺 $\mathrm{C} / \mathrm{C}$ 复合材料的拉伸强度为响应目标, 采用响 应曲面法构建针刺成型参数与针刺 $\mathrm{C} / \mathrm{C}$ 复合材料拉 伸强度的相关性数学模型, 分析针刺成型参数及其 交互作用对材料拉伸强度的影响趋势, 优化得到最 佳的拉伸强度, 并对模型的预测值进行验证。

\section{1 实验与模型构建}

\section{1 实验方法}

以针刺密度、针刺深度和网胎面密度为关键作 用因素, 采用 Design-expert 8.0.6 软件基于响应曲面 法设计了三因素、三水平、三个起点共 17 组预制体 针刺成型工艺试验 ${ }^{[18-20]}$ (见表 1)。其中, 预制体针刺 密度为 8 16 针 $/ \mathrm{cm}^{2}$, 深度为 $10 \sim 16 \mathrm{~mm}$, 网胎面密度 为 $50 \sim 150 \mathrm{~g} / \mathrm{m}^{2}$, 网胎碳纤维为 $\mathrm{T} 700$ 的 $12 \mathrm{~K}$ 短纤维。

按照表 1 所列的针刺成型参数, 在仿形芯模上 轴向铺放及纬向卷缠 12K T700 聚丙烯腈基无纬布, 并通过网胎针刺固定成型中空筒形件, 完成 17 件中 空筒形预制体针刺成型。中空筒形预制体尺寸为内 径 $232 \mathrm{~mm}$ 、高度 $150 \mathrm{~mm}$ 、厚度 $20 \mathrm{~mm}$ 。采用化学
气相渗透方法对筒形针刺预制体进行致密化, 得到 密度为 $1.64 \sim 1.66 \mathrm{~g} / \mathrm{cm}^{3}$ 的针刺 $\mathrm{C} / \mathrm{C}$ 复合材料筒形件。 沿 $\mathrm{C} / \mathrm{C}$ 复合材料筒形件轴向机加拉伸试样, 并在 SANS 5304-20KN 型万能试验机上按照 GBT14472005 标准测试 C/C 复合材料的拉伸强度, 相关结果 见表 1 。

\section{2 模型构建}

选择二次方程, 并考虑所有的二次项、一次项和 两两交叉项，获得二次响应曲面模型方程的通式为:

$$
Y=\beta_{0}+\sum_{i=1}^{3} \beta_{i} X_{i}+\sum_{i=1}^{3} \beta_{i i} X_{i} X_{i}+\sum_{i=1}^{3} \sum_{j=i+1}^{2} \beta_{i j} X_{i} X_{j}
$$

式中, $Y$ 为响应目标; $\beta_{0}$ 为截距; $\beta_{\mathrm{i}}$ 为一次模型系数; $\beta_{i i}$ 为二次模型系数; $\beta_{i j}$ 为交互作用系数; $X_{i}$ 为独立 变量。

应用 Design-expert8.0.6 实验设计软件的 BoxBehnken Design(BBD)方法, 以针刺成型参数为输入 相、拉伸强度为输出相(响应目标)进行数据处理 ${ }^{[21-23]}$, 拟合出回归方程。

\section{2 结果与讨论}

\section{1 响应曲面模型的显著性}

依表 1 各试验点数据, 应用回归分析确定一次 和二次相关系数, 所得拉伸强度 $(Y)$ 的响应曲面模型

表 1 预制体针刺参数及拉伸强度试验结果

Table 1 Needling parameters and tested tensile strengths of $\mathrm{C} / \mathrm{C}$ composites

\begin{tabular}{rrrrc}
\hline No. & $X_{1}$ & $X_{2}$ & $X_{3}$ & Tensile strength /MPa \\
\hline 1 & 8 & 10 & 100 & 89.80 \\
2 & 16 & 16 & 100 & 47.30 \\
3 & 12 & 13 & 100 & 87.56 \\
4 & 16 & 13 & 50 & 69.44 \\
5 & 12 & 10 & 150 & 86.72 \\
6 & 12 & 13 & 100 & 90.00 \\
7 & 16 & 13 & 150 & 83.70 \\
8 & 8 & 13 & 50 & 74.13 \\
9 & 16 & 10 & 100 & 72.83 \\
10 & 12 & 16 & 50 & 61.46 \\
11 & 12 & 13 & 100 & 79.66 \\
12 & 8 & 13 & 150 & 82.73 \\
13 & 12 & 13 & 100 & 93.10 \\
14 & 12 & 16 & 150 & 73.90 \\
15 & 12 & 13 & 100 & 80.73 \\
16 & 12 & 10 & 50 & 89.00 \\
17 & 8 & 16 & 100 & 70.96 \\
\hline
\end{tabular}

Note: $X_{1}, X_{2}$, and $X_{2}$ indicate needling density, needling depth and density of non-woven, respectively 
可表示为针刺密度 $\left(X_{1}\right)$ 、针刺深度 $\left(X_{2}\right)$ 和网胎面密度 $\left(X_{3}\right)$ 的二次多项式非线性回归方程:

$$
\begin{aligned}
Y= & 12.36146 X_{1}+19.25743 X_{2}-0.25087 X_{3}- \\
& 0.13927 X_{1} \cdot X_{2}+7.08125 \times 10^{-3} X_{1} \cdot X_{3}+ \\
& 0.024533 X_{2} \cdot X_{3}-0.52688 X_{1}^{2}-0.90653 X_{2}^{2}- \\
& 3.52500 \times 10^{-4} X_{3}^{2}-72.85340
\end{aligned}
$$

对回归方程及其系数进行显著性检验 ${ }^{[19-20]}$, 应用方 差法分析所拟合的响应曲面模型回归方程, 判定模 型的拟合效果：若模型显著性 $P \leqslant 0.05$, 说明模型拟 合显著; 若 $P \leqslant 0.01$, 则模型拟合极显著; 若 $P>0.05$ 时, 则模型拟合不显著。方程拟合性质由复相关系 数平方和(R-Squared)表达, 其越趋近于 1 , 说明该 方程的预测效果越好。

响应曲面模型回归方程及其系数的显著性分 析结果列于表 2 。由于模型 $P=0.0206<0.05$, 表明该 模型拟合显著, 模型失拟性检验无显著性差异 (失 拟度 $=0.2027>0.05)$, 且方程复相关系数平方和 $(R-$ Squared)为 0.8695 , 说明实际验证模拟值与实测值 吻合程度较高, 模型拟合效果较好, 拟合模型能够 反映试验结果, 可较为准确反映针刺成型参数对 $\mathrm{C} / \mathrm{C}$ 复合材料拉伸性能影响规律, 较好地分析和预 测 $\mathrm{C} / \mathrm{C}$ 复合材料的拉伸性能。

从表 2 回归方程系数的显著性分析结果可以 看出, 针刺深度对响应目标的影响极为显著, 针刺 密度及其二次项、针刺深度二次项对响应目标的影 响显著。其中, 针刺密度、针刺深度和网胎面密度 对拉伸强度的影响显著性依次为针刺深度 $>$ 针刺 密度 $>$ 网胎面密度, 两两交互作用的影响显著程
度为针刺深度和网胎面密度的交互作用 $>$ 针刺深 度和针刺密度的交互作用 $>$ 针刺密度和网胎面密 度的交互作用。

\section{2 针刺参数对拉伸强度的影响}

以回归方程为数学模型绘制的响应曲面图可形 象描绘拉伸性能与各个针刺成型参数的内在关系。 图 1 3 为针刺密度、针刺深度、网胎面密度两两交 互作用时，拉伸强度的响应曲面及等高线图，由这 些图可以获得任一针刺参数下的拉伸强度响应值, 分析各针刺参数及其交互作用对拉伸强度的影响趋 势，并确定最佳取值范围。

图 1 为针刺密度、针刺深度交互作用拉伸强度 响应曲面及等高线图, 可以看出, 在给定网胎面密 度下, 针刺密度、针刺深度交互作用时的响应曲面 呈现凸曲面, 说明随着针刺密度、针刺深度的增大, 拉伸强度先提高后降低, 这主要是由于针刺预制体 增强特性决定的 ${ }^{[13-17]}$ 。

针刺预制体是通过刺针倒钩引入贯穿多层的 Z 向纤维束, 形成垂直的“销钉”结构, 整体上构成类 似于“钉板”的三维网状结构, 起到层间增强作用, Z 向纤维束来自于网胎层无序碳纤维, 并与网胎层无 序碳纤维相互缠结, 使针刺预制体各层间紧密结合, 纤维间的缠结度越高, 预制体密实度就越高, 最终 预制体整体纤维体积分数就越高, 从而赋予预制体 较高的整体性能。复合材料拉伸强度主要由增强纤 维的强度和沿载荷方向纤维的体积分数来控制, 在 $\mathrm{C} / \mathrm{C}$ 复合材料中, 碳纤维作为主要承载件承受大部

表 2 针刺 $\mathrm{C} / \mathrm{C}$ 拉伸强度响应曲面模型回归方程及相关系数显著性分析表

Table 2 Analysis of signification for response surface model and its correlation coefficient of tensile strength

\begin{tabular}{crrrrr}
\hline Source & Sum of squares & df & Mean square & $F$ Value & $P$-value prob $>F$ \\
\hline Model & 1978.209000 & 9 & 219.800900 & 5.180009 & 0.0206 \\
$X_{1}$ & 245.865300 & 1 & 245.865300 & 5.794263 & 0.0470 \\
$X_{2}$ & 897.290700 & 1 & 897.290700 & 21.146290 & 0.0025 \\
$X_{3}$ & 136.248800 & 1 & 136.248800 & 3.210950 & 0.1163 \\
$X_{1} X_{2}$ & 11.172310 & 1 & 11.172310 & 0.263296 & 0.6237 \\
$X_{1} X_{3}$ & 8.023056 & 1 & 8.023056 & 0.189078 & 0.6768 \\
$X_{2} X_{3}$ & 54.169600 & 1 & 54.169600 & 1.276605 & 0.2958 \\
$X_{1}^{2}$ & 299.220600 & 1 & 299.220600 & 7.051679 & 0.0327 \\
$X_{2}^{2}$ & 280.274500 & 1 & 280.274500 & 6.605179 & 0.0370 \\
$X_{3}^{2}$ & 3.269901 & 1 & 3.269901 & 0.077061 & 0.7893 \\
Residual & 297.027800 & 7 & 42.432540 & - & - \\
Lack of Fit & 192.536200 & 3 & 64.178730 & 2.456799 & - \\
Pure Error & 104.491600 & 4 & 26.122900 & - & - \\
Cor Total & 2275.236000 & 16 & - & - & - \\
$R$-Squared & 0.869500 & - & - & - & - \\
Adj $R$-Squared & 0.701600 & - & - & -
\end{tabular}



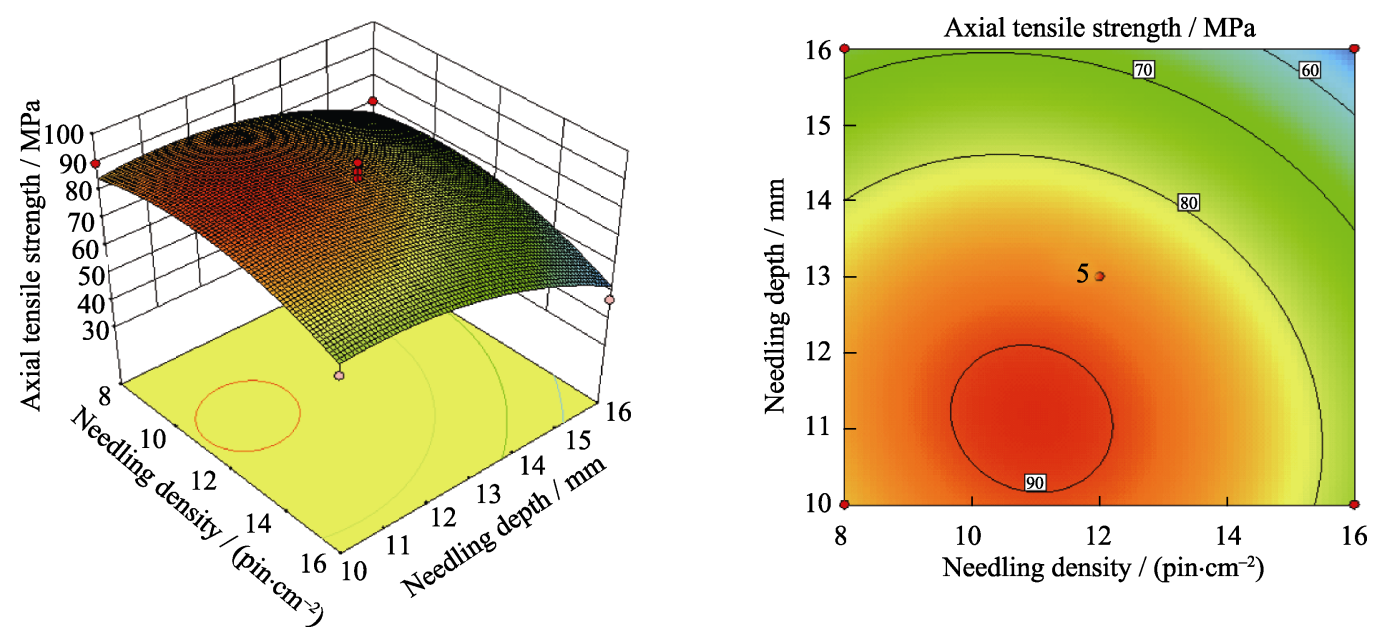

图 1 针刺密度、针刺深度交互作用拉伸强度响应曲面及等高线图(网胎面密度 $100 \mathrm{~g} / \mathrm{m}^{2}$ )

Fig. 1 Response surface and contour of tensile strength influenced by the interaction of needling density and needling depth (The density of non-woven is $100 \mathrm{~g} / \mathrm{m}^{2}$ )
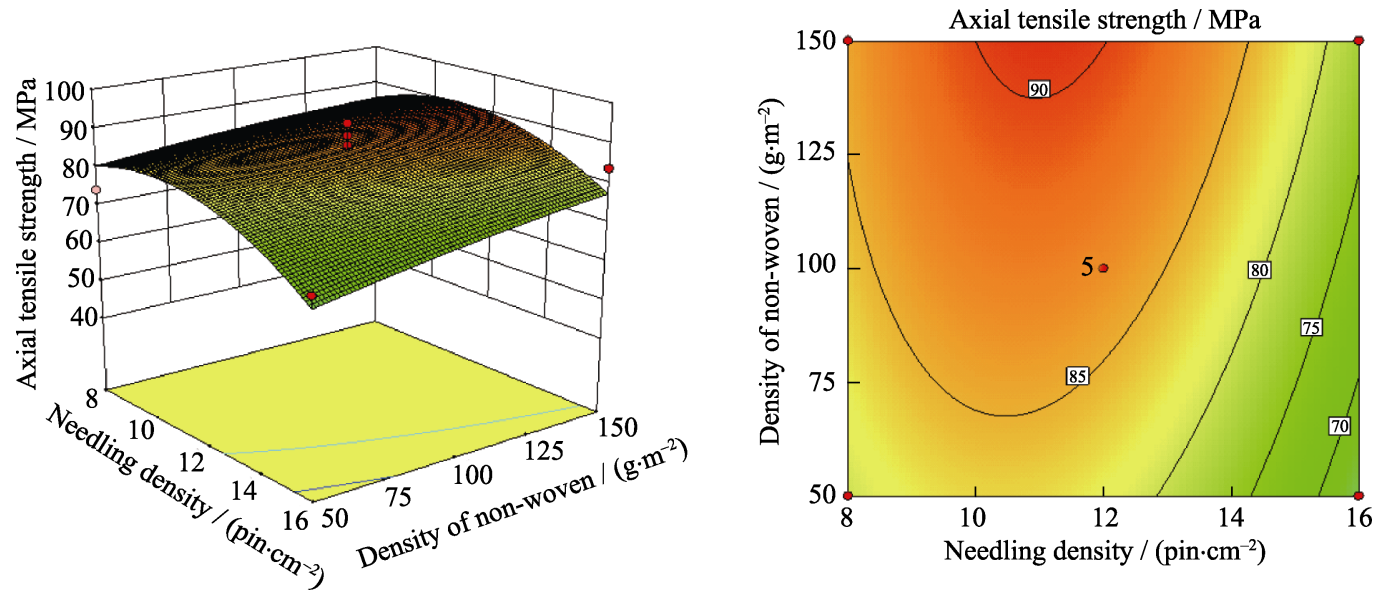

图 2 针刺密度、网胎面密度交互作用拉伸强度响应曲面及等高线图(针刺深度 $13 \mathrm{~mm}$ )

Fig. 2 Response surface and contour of tensile strength influenced by the interaction of needling density and density of non-woven (The needling depth is $13 \mathrm{~mm}$ )
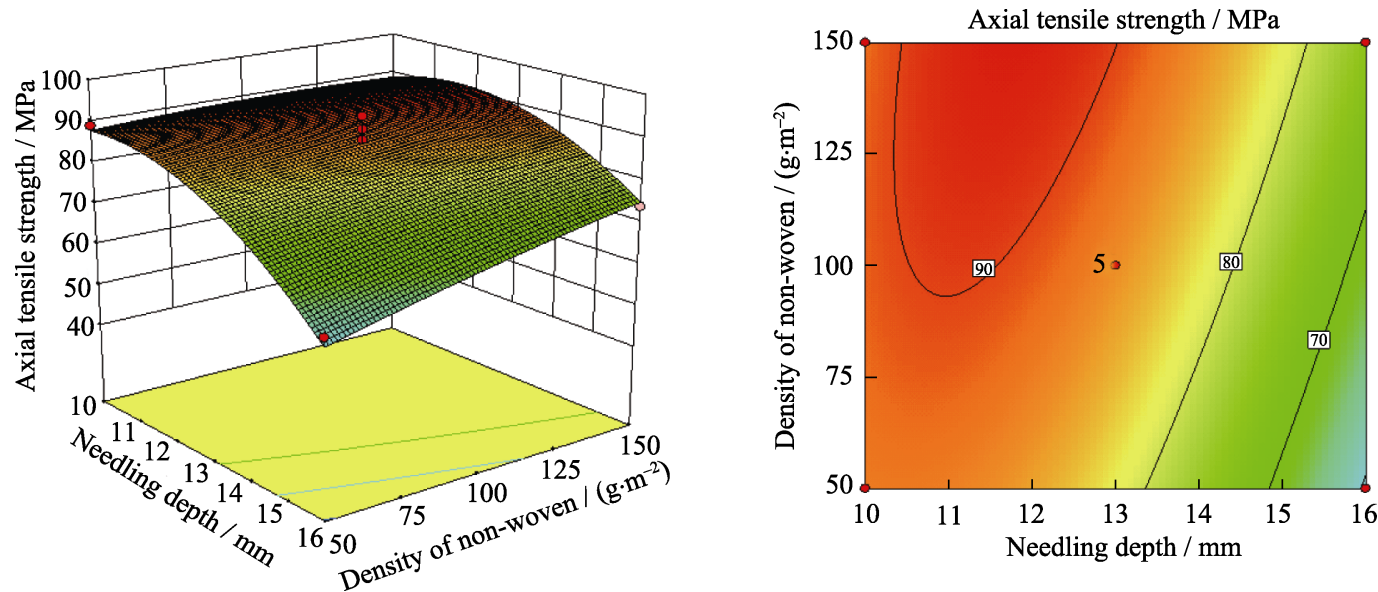

图 3 针刺深度、网胎面密度交互作用拉伸强度响应曲面及等高线图(针刺密度 $12 \mathrm{pin} / \mathrm{cm}^{2}$ )

Fig. 3 Response surface and contour of tensile strength influenced by interaction of needling depth and density of non-woven (The needling density is $12 \mathrm{pin} / \mathrm{cm}^{2}$ )

分应力, 一般认为纤维体积分数越高则其 $X Y$ 向拉 伸强度越高。由于在针刺预制体成型过程中, 引入 Z
向短纤维，刺针会对 $X Y$ 向碳布纤维、XY 向网胎短 纤维以及已经引入的 Z 向短纤维造成不同程度的损 
伤, 纤维长度会降低, 导致预制体强度降低, 因此 针刺成型参数在不同取值区间内对纤维缠结度、预 制体密实度及纤维损伤影响程度决定了不同阶段拉 伸强度随针刺参数的变化趋势。

图 2 为针刺深度恒定时, 针刺密度、网胎面密 度交互作用下材料拉伸强度响应面及等高线图。图 2 表明, 针刺密度对拉伸强度的影响不是单一的, 这是由于预制体密实度与 $X Y$ 向碳纤维损伤两方面 对拉伸强度不同影响趋势共同作用的结果。当针刺 密度较小时, 针刺过程对纤维的损伤较小, 随着针 刺密度的增加, Z 向纤维束数目提高, 纤维间缠结度 与针刺预制体密实度相应提高, 这阶段密实度提高 对拉伸强度的增强作用大于纤维损伤对拉伸强度的 不利影响，预制体拉伸强度总体呈现上升。当针刺 密度继续增大到一定程度时, 一方面随着针孔数增 多, 对 $\mathrm{XY}$ 向纤维的损伤加剧, $\mathrm{XY}$ 向纤维长度下降 明显, 从而影响预制体的拉伸强度 ${ }^{[13-14]}$; 另一方面, $\mathrm{Z}$ 向纤维的损伤也会导致短纤维长度下降, 对短纤 维的缠结度及针刺预制体的整体紧密度也有不利影 响，因此预制体拉伸强度总体呈现下降。

图 3 为针刺密度一定时针刺深度、网胎面密度 交互作用下材料拉伸强度的响应面及等高线图, 可 以看出, 针刺深度对拉伸强度的影响也不是单一的, 其变化仍然是针刺深度对预制体增强与损伤作用的 综合结果, 并受刺穿各层的刺针钩槽的形状、大小 和位置影响显著。针刺深度对拉伸强度增强作用主 要体现在针刺深度增大, 引入的 $\mathrm{Z}$ 向纤维长度增大, 同时刺针上能够抓取短纤维的钩槽数增多, 相应引 入的 $\mathrm{Z}$ 向纤维数增多, 从而提高纤维间缠结度, 针 刺预制体密实度也相应提高, 对拉伸强度具有显著 影响。

针刺深度增大对于 XY 向纤维的损伤与针刺密 度不同。针刺密度的增加导致刺孔稳定增加, 对 $X Y$ 向纤维的损伤随之稳定增大, $\mathrm{XY}$ 向纤维长度随之 稳定减小, 从而稳定影响拉伸强度, 在图 2 响应曲 面图上呈现相对平缓的变化态势。针刺深度的增加 对 XY 向纤维的损伤影响程度取决于刺穿单元层(一 层无纬布与一层网胎构成一个单元层)的刺针钩槽 的形状、大小和位置, 由于针刺是逐层进行, 针刺深 度变化对 XY 向纤维的损伤度呈现如下特征: (1)在 低于 $11 \mathrm{~mm}$ 的针刺深度下, 参与抓取短纤维的刺针 钩槽少且为定数 (到一定深度, 下一组钩槽才参与), 引入的 Z 向纤维数及对纤维的损伤变化不大, 影响 预制体密实度的主要为引入的 $\mathrm{Z}$ 向纤维长度, 因此 预制体拉伸强度稳步增加; (2)在 11 13 $\mathrm{mm}$ 的针刺 深度范围内, 随着针刺深度的增加, 参与抓取短纤
维的刺针钩槽数显著增多, 引入的 $\mathrm{Z}$ 向纤维数及对 纤维的损伤度都会发生较大变化, 拉伸强度变化显 著, 其变化的趋势起决于 $\mathrm{Z}$ 向纤维数增大引起预制 体密实度提高与纤维损伤对拉伸强度共同作用。此 阶段 Z 向纤维数随着钩槽的间断加入, 发生较为明 显的增加, 有利于纤维缠结度、预制体密实度的提 高, 另一方面由于针板上相邻刺针间纤维断裂损伤 逐渐加大, 引入的 $\mathrm{Z}$ 向纤维长度变化将会出现反转, 导致纤维缠结度、预制体密实度的降低，响应面上拉 伸强度呈现先升后降的变化表明此阶段损伤逐步起 主导作用。(3)在大于 $13 \mathrm{~mm}$ 的针刺深度下, 刺针钩 槽贯穿的单元层数相对增多, 相当于每个单元层实 际受到的针刺次数增多, 相对于较低针刺深度, 其 对纤维的损伤相当于针刺密度与针刺深度交互作用 损伤的叠加，对于拉伸强度的影响起到增大效应。

图 2 与图 3 对比结果表明, 针刺深度对拉伸强 度的影响显著度比针刺密度高。尤其是当网胎面密 度恒定时, 可引入的 $\mathrm{Z}$ 向纤维数受限, 针刺深度对 $X Y$ 向纤维损伤叠加会引起拉伸强度明显下降。图 3 中网胎面密度在较小的区间(50 75 $\left.\mathrm{g} / \mathrm{cm}^{2}\right)$, 针刺深 度增大至 $13 \mathrm{~mm}$ 左右以上时, 拉伸强度开始陡然下 降证实了这一变化。当网胎面密度在较高的区间 $\left(125 \sim 150 \mathrm{~g} / \mathrm{cm}^{2}\right)$, 针刺深度 $14 \mathrm{~mm}$ 左右, 拉伸强度 仍然缓慢下降, 这是由于较高的网胎面密度, 可引 入的 Z 向纤维数相对增多, 对提高预制体密实度有 利, 同时随网胎面密度的增加, 网胎本身的强度也 相应增加, 提高了预制体与针刺 $\mathrm{C} / \mathrm{C}$ 的拉伸强度, 缓解了纤维损伤所引起的针刺 $\mathrm{C} / \mathrm{C}$ 拉伸强度的下降 趋势, 使之变化趋于平缓。

图 2 和图 3 的对比结果进一步表明, 针刺密度 与网胎面密度的交互作用、针刺深度与网胎面密度 的交互作用对拉伸强度的影响趋势相同, 只是程度 不同, 针刺深度与网胎面密度的交互作用相对显著, 较高的拉伸强度响应值都处于低针刺深度区域、低 针刺密度区域、高网胎面密度区域。

\section{3 优化与预测}

表 3 为响应曲面回归方程拟合值与实际值的对 照表，最大相对误差 $10.82 \%$, 说明对于针刺 C/C 复 合材料, 此模型的预测值较为准确。

基于式(2)求取参数范围内的极值和极值点得到 表 4: 针刺密度为 $11.17 \mathrm{pin} / \mathrm{cm}^{2}$, 针刺深度为 $11.79 \mathrm{~mm}$ 且网胎面密度为 $150 \mathrm{~g} / \mathrm{m}^{2}$ 时, 拉伸强度最大, 达 $91.87 \mathrm{MPa}$; 针刺密度为 $16 \mathrm{pin} / \mathrm{cm}^{2}$, 针刺深度为 $16 \mathrm{~mm}$ 且网胎面密度为 $50 \mathrm{~g} / \mathrm{m}^{2}$ 时, 拉伸强度最小, 达 $42.31 \mathrm{MPa}$ 。 
表 3 针刺 $\mathrm{C} / \mathrm{C}$ 复合材料拉伸强度响应面拟合值 与实际值的对照表

Table 3 Comparison of the fitting values of the fitting equation of response surface with the actual values

\begin{tabular}{ccrc}
\hline Actual value & Predicted value & Residual & Relative error \\
\hline 89.80 & 84.684375 & 5.115625 & $5.70 \%$ \\
72.83 & 76.939375 & -4.114375 & $5.65 \%$ \\
70.96 & 66.845625 & 4.114375 & $5.80 \%$ \\
47.30 & 52.415625 & -5.115625 & $10.82 \%$ \\
74.13 & 80.331875 & -6.201875 & $8.37 \%$ \\
69.44 & 66.411875 & 3.028125 & $4.36 \%$ \\
82.73 & 85.753125 & -3.028125 & $3.66 \%$ \\
83.70 & 77.498125 & 6.201875 & $7.41 \%$ \\
89.00 & 87.913750 & 1.086250 & $1.22 \%$ \\
61.46 & 59.372500 & 2.087500 & $3.40 \%$ \\
86.72 & 88.807500 & -2.087500 & $2.41 \%$ \\
73.90 & 74.986250 & -1.086250 & $1.47 \%$ \\
90.00 & 86.810000 & 3.190000 & $3.54 \%$ \\
87.56 & 86.810000 & 0.750000 & $0.86 \%$ \\
82.66 & 86.810000 & -4.150000 & $5.02 \%$ \\
80.73 & 86.810000 & -6.080000 & $7.53 \%$ \\
93.10 & 86.810000 & 6.290000 & $6.76 \%$ \\
\hline & & &
\end{tabular}

表 4 拉伸强度取值范围

Table 4 Scope of tensile strength

\begin{tabular}{ccccc}
\hline & $\begin{array}{c}\text { Needling } \\
\text { density } \\
/\left(\text { pin } \cdot \mathrm{cm}^{-2}\right)\end{array}$ & $\begin{array}{c}\text { Needling } \\
\text { depth/mm }\end{array}$ & $\begin{array}{c}\text { Density of } \\
\text { non-woven } \\
/\left(\mathrm{g} \cdot \mathrm{m}^{-2}\right)\end{array}$ & $\begin{array}{c}\text { Tensile } \\
\text { strength } \\
/ \mathrm{MPa}\end{array}$ \\
\hline Max & 11.17 & 11.79 & 150.00 & 91.87 \\
Min & 16.00 & 16.00 & 50.00 & 42.31 \\
\hline
\end{tabular}

表 5 拉伸强度优化结果

Table 5 Optimization results of tensile strength

\begin{tabular}{|c|c|c|c|c|c|}
\hline \multirow{2}{*}{$\begin{array}{l}\text { Needling } \\
\text { density } \\
/\left(\operatorname{pin} \cdot \mathrm{cm}^{-2}\right)\end{array}$} & \multirow{2}{*}{$\begin{array}{c}\text { Needling } \\
\text { depth } \\
\text { /mm }\end{array}$} & \multirow{2}{*}{$\begin{array}{c}\text { Density of } \\
\text { non-woven } \\
/\left(\mathrm{g} \cdot \mathrm{m}^{-2}\right)\end{array}$} & \multicolumn{2}{|c|}{$\begin{array}{l}\text { Tensile strength/ } \\
\text { MPa }\end{array}$} & \multirow{2}{*}{$\begin{array}{l}\text { Relative } \\
\text { error }\end{array}$} \\
\hline & & & $\begin{array}{c}\text { Predictive } \\
\text { value }\end{array}$ & $\begin{array}{l}\text { Actual } \\
\text { value }\end{array}$ & \\
\hline 11 & 12 & 50 & 86.72 & 83.53 & $3.68 \%$ \\
\hline 11 & 11 & 50 & 88.62 & 90.71 & $2.36 \%$ \\
\hline 12 & 11 & 50 & 87.69 & 85.23 & $2.28 \%$ \\
\hline 12 & 12 & 50 & 85.65 & 88.64 & $3.49 \%$ \\
\hline
\end{tabular}

在实际应用中，基于预制体成型工艺性，针刺 密度和针刺深度取整数, 并通常采用低网胎面密度,

而且根据显著性分析, 网胎面密度对拉伸强 度影响为非显著, 因此在表 4 中得出的针刺密度 $11.17 \mathrm{pin} / \mathrm{cm}^{2}$ 、针刺深度为 $11.79 \mathrm{~mm}$ 附近取整, 并 采用 $50 \mathrm{~g} / \mathrm{m}^{2}$ 网胎面密度, 其结果如表 5 。其中以针刺 密度 $11 \mathrm{pin} / \mathrm{cm}^{2}$ 、针刺深度 $11 \mathrm{~mm}$ 、网胎面密度 $50 \mathrm{~g} / \mathrm{m}^{2}$ 为工艺参数进行设计, 对应预测值为 $88.62 \mathrm{MPa}$, 实 际验证值为 $90.71 \mathrm{MPa}$, 相对误差 $2.36 \%$, 工艺和性 能都得到优化。

\section{3 结论}

1) 应用响应曲面法构建了针刺成型参数与针 刺 $\mathrm{C} / \mathrm{C}$ 复合材料拉伸强度的响应曲面模型，显著性 分析表明, 该模型的 $P=0.0206$, 失拟性检验无显 著性差异 (失拟度 $=0.2027>0.05$ ), 且复相关系数平 方和 $(R$-Squared)为 0.8695 , 拟合的模型能够反映试 验结果, 其预测值与实际值最大误差为 $10.82 \%$, 可 以较好地对针刺 $\mathrm{C} / \mathrm{C}$ 复合材料拉伸强度进行分析和 预测。

2) 针刺深度对拉伸强度有极显著的影响, 针刺 密度、针刺密度的二次项、针刺深度的二次项对拉 伸强度有显著影响, 针刺深度、针刺密度对拉伸强 度的影响为先升后降, 其变化趋势受刺针钩槽的影 响较大。

3) 在本针刺参数取值范围内, 拉伸强度的预测 区间为 42.31 91.87 MPa，模型优化出的针刺成型参 数为: 针刺密度 $11 \mathrm{pin} / \mathrm{cm}^{2}$ 、针刺深度 $11 \mathrm{~mm}$ 、网胎 面密度 $50 \mathrm{~g} / \mathrm{m}^{2}$, 相应拉伸强度预测值为 $88.62 \mathrm{MPa}$, 实际验证值为 $90.71 \mathrm{MPa}$, 相对误差 2.36\%。

\section{参考文献:}

[1] FITZER E, MANOCHA L M. Carbon Reinforcements and Carbon/Carbon Composites. Germany: Springer-verlag Berlin Heidelberg GmbH\&Co.K, 1998: 71-95.

[2] MOURITZ A P, BANNISTER M K, FALZON P J, et al. Review of applications for advanced three-dimensional fibre textile composites. Composites: Part A, 1999, 30: 1445-1461.

[3] FAWCETT R, HORNICK J, BACKLUND D, et al. Advanced $3^{\text {rd }}$ Stage Carbon-Carbon Exit Cone. AIAA 2008-4888, 2008.

[4] CHEN XIAO-MING, CHEN LI, ZHANG CHUN-YAN, et al. Three-dimensional needle-punching for composites - A review. Composites: Part A, 2016, 85: 12-30.

[5] RUSSELL A ELLIS. Testing of Novoltex 3D Carbon-Carbon Integral Throat and Cones (ITECs). AIAA-88-3361, 1988.

[6] MONTAUDON M. Arine 5 Carbon-Carbon Nozzle Throat: Development Result. AIAA95-3016, 1995.

[7] BROQUERE B H. Carbon/Carbon Nozzle Exit Cones: SEP's Experience and New Development. AIAA97-2674, 1997.

[8] BOURY D, FILIPUZZL L. Sepcarb Materials For Solid Rocket Booster Nozzle Components. AIAA 01-3438, 2001.

[9] LACOMBE A, PICHON T, Lacostet M. 3D Novoltex and Naxeco Carbon-Carbon Nozzle Extensions: Matured, Industrial and Available Technologies to Reduce Programmatic and Technical Risks and to Increase Performance of Launcher Upper Stage Engines. AIAA 2008-5236, 2008.

[10] LACOMBE A, PICHON T, LACOSTET M. 3D Carbon-Carbon Composites are Revolutionizing Upper Stage Liquid Rocket Engine Performances by Allowing Introduction of Large Nozzle Extension. AIAA 2009-2678, 2009.

[11] SU JUN-MING, XIAO ZHI-CHAO, LIU YONG-QIONG, et al. 
Preparation and characterization of carbon/carbon aircraft brake materials with long service life and good frictional properties. New Carbon Materials, 2010, 25(5): 329-334.

[12] SU JUN-MING, ZHOU SHAO-JIAN, LI RUI-ZHEN, et al. A rewiew of carbon-carbon composites for engineering applications. New Carbon Materials, 2015, 30(2): 107-114.

[13] LI FEI, LIU JIAN-JUN, CHENG WEN, et al. Effect of technology parameters on mechanical performances of $\mathrm{C} / \mathrm{C}$ composites needling fabrication. Carbon, 2004, (1): 25-27.

[14] JI A-LIN, CUI HONG, LI HE-JUN, et al. Perfomance analysis of a carbon cloth/felt layer needled preform. New Carbon Materials. 2011, 26(2): 11-18.

[15] JI A-LIN, LI HE-JUN, CUI HONG, et al. Axial thermodynamic performance analysis of the different preform C/C composites. Journal of Inorganic Materials, 2010, 09(25): 995-998.

[16] ZHANG XIAO-HU, LI HE-JUN, HAO ZHI-BIAO, et al. Influence of needle-punching processing on mechanical properties of $\mathrm{C} / \mathrm{C}$ composites reinforced by carbon cloth and carbon fiber net. Journal of Inorgnic Materials. 2007, 22(5): 963-967.

[17] SU JUN-MING, CUI HONG, LI RUI-ZHEN, et al. The structure and property of new needle carbon cloth $\mathrm{C} / \mathrm{C}$ composite. New Carbon Materials, 2000, 15(2): 11-15.
[18] 徐向宏, 何明珠. 试验设计与 Design-Expert、SPSS 应用. 北京: 科学出版社, 2010: 146-157.

[19] GEORGIOU S D, STYLIANOU S, AGGARWAL M. A class of composite designs for response surface methodology. Computational Statistics and Data Analysis, 2014, 71: 1124-1133.

[20] ADELEKE A O, LATIFF A B AZIZ A B, AL-GHEETHI A A, et al. Optimization of operating parameters of novel composite adsorbent for organic pollutants removal from POME using response surface methodology. Chemosphere, 2017, 174: 232-242.

[21] THAKRE A A, SONI S. Modeling of burr size in drilling of aluminum silicon carbide composites using response surface methodology. Engineering Science and Technology, 2016, 19: 11991205.

[22] BOROOJ M BAGHERY, SHOUSHTARI M AHMAD, HAJI AMINODDIN, et al. Optimization of plasma treatment variables for the improvement of carbon fibres/epoxy composite performance by response surface methodology. Composites Science and Technology, 2016, 128: 215-221.

[23] XU YING, LI MING-LI, ZHAO XUAN-MIN, et al. The response curved surface regression analysis technique - the application of a new regression analysis technique in materials research. Rare Metal Materials and Engineering, 2001, 30(6): 428-432. 